\title{
Optical characterization of ethane droplets in the vicinity of critical pressure
}

\author{
Chanisa Kanjanasakul ${ }^{1}$, Frédéric Grisch ${ }^{1}$, Sawitree Saengkaew ${ }^{2}$, and Gérard Gréhan ${ }^{1, *}$ \\ ${ }^{1}$ UMR CNRS 6614/CORIA, Université de Rouen, Avenue de l'Université, 76800 Saint Etienne du Rouvray, France \\ ${ }^{2}$ RainbowVision, 30 rue Eau de Robec, 76000 Rouen, France
}

Received: 2 April 2019 / Accepted: 25 May 2020

\begin{abstract}
The characterization of the properties of fuel droplets around the critical pressure is an experimental challenge. The aim of this study is to measure the size and the refractive index of droplets in high pressure conditions, close to the ones of the critical point. For this purpose, an experimental facility of injection of quasi-monodispersed droplets at high pressure has been developed. Rainbow refractometry has been used to probe droplets in the pressure range 1-50 bar. For water and ethanol droplets, evolutions of their index of refraction with pressure were obtained. For measurements with a liquid fluid near its critical point, ethane was selected because its critical point is 48.7 bar and $32.2^{\circ} \mathrm{C}$. Refractive index measurements on ethane droplets were carried out in the range of pressure and temperature $40-46$ bar and $18-25{ }^{\circ} \mathrm{C}$ respectively. These unique results show that the rainbow refractometry diagnosis offers undeniable potentials for studying fuel injection under transcritical thermodynamic conditions.
\end{abstract}

\section{Introduction}

Internal combustion engines play an important role in the fields of power, propulsion and energy. During the last decades, huge efforts were accomplished to develop advanced engine technologies with the potential for significantly improved liquid fuel injection into the engine cylinder as a spray. As everybody knows, fuel injection is a key controlling factor for engine performances and emissions but this one adds significant degrees of freedom and complexity to the designed optimization process. Up to now, lack of accurate models for spray injection still hinders the design of optimized, clean, high efficiency engine technologies. This is particularly the case for many advanced internal combustion engines in which cylinder pressures exceed the thermodynamic critical pressure of the fuel at the start of injection. In that case, the classical view of spray atomization and second breakup processes comes into question. Indeed, fuel injection occurs at "transcritical" conditions, where the fuel is supercritical with respect to the pressure and subcritical with respect to temperature. Under such conditions, substantial thermodynamic non-idealities and transport anomalies appear. Mixture properties exhibit liquid-like densities, gas-like diffusivities, and pressure-dependent solubilities. The isothermal compressibility and constant pressure-specific heat increase significantly while the heat of vaporization and the surface tension is reduced [1]. This combination of

\footnotetext{
* Corresponding author: grehan@coria.fr
}

properties alters in-cylinder mixing and combustion processes and must be rigorously understood. Treating these processes also has direct relevance to the use of multicomponent fuels such as gasoline or diesel fuels. To move beyond empirically based spray models, experiments must be performed in situations in which a complete description of these flows over a wide range of thermodynamic conditions can be provided. In particular, the knowledge of properties of fuel droplets must be known in terms of size and temperature of droplets for validating evaporation and mixing processes that govern combustion. Quantitative measurement techniques are then necessary to be developed for probing such processes and then providing a complete temporal and spatial history of fuel properties during injection. Until now, current diagnostics for droplet sizes are fairly mature, but are limited to specific drop sizes ranges and fluids in which pressure is not elevated (few bars). Similarly, measurements of temperature of droplets can be performed using Global Rainbow Refractometry (GRR) but its application is actually only restricted to pure fuel sprays injected at atmospheric pressure. To characterize fuel droplets under transcritical conditions, a focus on the extension of the application of the GRR technique on moving droplets under high-pressure conditions was investigated. Rainbow refractometry is a laser-based light scattering measurement technique that determines the refractive index of an individual droplet. The temperature of a droplet is then determined from the refractive index from a relationship between the refractive index and the temperature. Until 
now, all previous refractive index measurements of liquids under high-pressure conditions were only performed on liquids contained in high pressure optical cells but never directly on free-falling droplets [2-8]. For that, the GRR technique is selected as it is able to measure refractive indices on moving small droplets.

Our intent in this paper is to provide further insight into the capabilities and limitations of the GRR. Specifically, the maximum theoretical errors in the refractive-index temperature measurements on moving droplets under high pressure conditions, still being in subcritical condition, were studied. This article is structured as follows: Section 2 provides a basic background on properties of fluids in subcritical and critical states as well as a detailed description of the fundamentals of the GRR laser diagnostic. Section 3 describes the experimental setup developed in the current study. Section 4 shows the results on measurements of the refractive index measurements on free-falling water and ethanol droplets under high pressure conditions, demonstrating the capabilities of application of the rainbow refractometry in such extreme conditions. Section 4 presents the measurements on free-falling liquid ethane droplets at pressures close to its critical pressure. Conclusions of this study are given in Section 5 .

\section{Background}

\subsection{Supercritical fluid}

A fluid is in its supercritical state once its temperature $T$ exceeds its critical temperature $\left(T_{\mathrm{cr}}\right)$ and once at the same time its pressure $P$ exceeds its critical pressure $\left(P_{\text {cr }}\right)[9]$. The Pressure-Temperature $(P T)$ phase diagram in Figure 1 classifies states, at which the fluid is solid, liquid, gaseous or supercritical. Note on this diagram that there is no phase transition, when a fluid is compressed isothermally from the gas phase to the supercritical state or when a fluid is heated isobaric from the liquid phase to the supercritical state. From the $P T$ phase diagram, the red line defines the sublimation curve denoting the transition between vapor and solid phases. The endpoint of this line is the triple point at which a substance co-exists as a gas, a liquid and a solid. The green line is the fusion curve representing the transition between solid and liquid phases. The blue line is the vaporization curve that differentiates the gaseous from the liquid regime and according to Gibb's rule defines the couples of pressure and temperature at which a liquid and a gaseous phases of a substance co-exist. Finally, the critical pressure and the critical temperature define the endpoint of the vapor-liquid equilibrium (i.e. vaporization curve). In order to understand the properties at the critical point, it is interesting to visualize the modification of the properties of a pure substance contained in a closed chamber when the vaporization curve is followed from the triple point to the critical point. At the triple point, coexistence of a solid phase, liquid phase and gaseous phase is noted. Moving away from the triple point to the critical point, the solid phase will disappear. The remaining liquid and gas phases will be split, which is visible by the existence of a meniscus forming between the two phases. The liquid

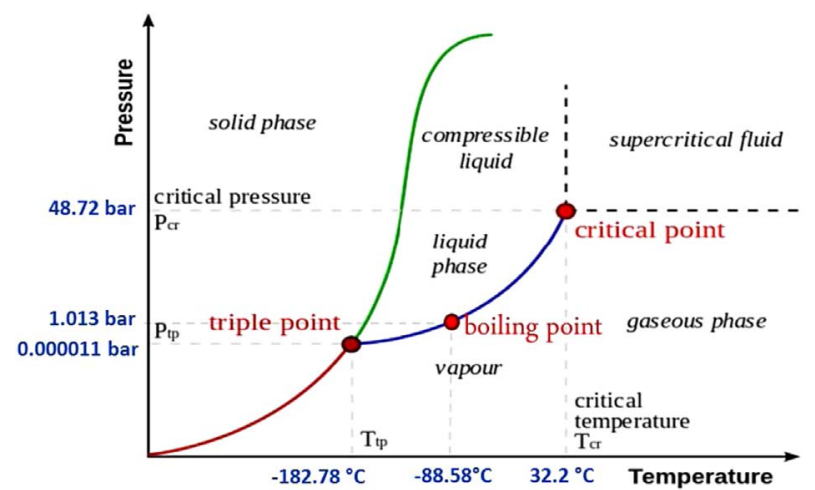

Fig. 1. Pressure-temperature phase diagram of pure substances. The values of pressure and temperature for ethane are also given [1].

phase is then much denser than the gas phase and it will accumulate at the bottom of the chamber, while the light gas phase will fill the remaining volume of the chamber on top of the dense liquid phase. Moving nearer to the critical point goes along with a density increase in the gas phase, while the density of the liquid remains relatively similar. At the same time, the meniscus separating the dense liquid from the gas phase will be weaker. This merely reflects the view that the properties (including the density) of the liquid and the gas phases converge as the critical point is approaching. At the critical point, the "liquid" and the "gas" phases are finally the same. Hence, the physical distinction between liquid and vapor represented by the meniscus disappears and the whole chamber is then filled with a fluid characterizing only one phase. Above the critical point, the fluid is termed a supercritical fluid in which the liquid and gaseous phases cannot be distinguished.

As the aim of this study is to probe liquid droplets of a pure substance in thermodynamic conditions ranging from subcritical to supercritical conditions, ethane $\left(\mathrm{C}_{2} \mathrm{H}_{6}\right)$ was selected because this molecule offers attractive thermodynamic conditions for easily reproducing the critical state conditions in laboratory experiments. Indeed, the critical point of ethane is at a pressure of 48.72 bar and a temperature of $32.17^{\circ} \mathrm{C}$. These values are not elevated compared to those of water in which a critical pressure of 221 bar and critical temperature of $374{ }^{\circ} \mathrm{C}$ are noted. For a better clarity, its values of pressure and temperature for the different ethane thermodynamic states have been reported in the $P T$ phase diagram displayed in Figure 1.

\subsection{Rainbow refractometry diagnostic}

Rainbow refractometry is one of the optical measurement diagnostics that provides an ability to determine the refractive index of individual droplets. The background physics of this technique is similar to the rainbow phenomenon observed in the sky as shown in Figure 2. In 1637, René Descartes was the first to introduce a physical explanation of the rainbow phenomenon considering the light passing through an individual droplet [10]. For a basic explanation, 


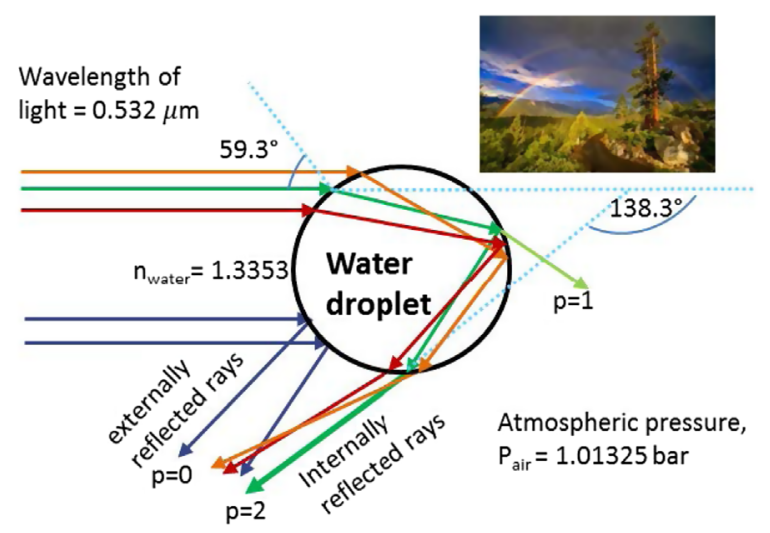

Fig. 2. First three-orders of light scattering from a droplet of water.

the parallel rays of light impinging on a homogeneous spherical droplet are partially externally reflected and partially refracted inside the droplet. Reflection of light inside the droplet can also occur. After a given number of internal reflections, the light rays are refracted once more when exiting from the droplet. The rainbow pattern is located at the extremum deviation of these rays. The primary and secondary rainbows are denoted as the extremum of deviation of light rays having one internal reflection or two internal reflections, respectively. Figure 2 shows the path of light rays having one internal reflection and the light reflected at the external surface of a water droplet. They are denoted as the internally reflected ray and the externally reflected ray, respectively. Using the principles of geometrical optics, it might be useful to denote the light rays leaving the particle with the van de Hulst's notation [11]:

- $p=0$ for the externally reflected ray,

- $p=1$ for the ray refracted twice,

- $p=2$ for the ray experimencing one internal reflection and two refractions (the internally reflected ray),

- $p=3$ for the ray experimencing two internal reflections and two refractions,

- $p=k$ for the ray experimencing $k-1$ internal reflections and two refractions.

In the sky, the colour of the primary and the secondary rainbows due to chromatic dispersion of light in water droplets can be observed. The area between the primary and the secondary rainbows is called Alexander's dark band where the intensity is very small in comparison with primary and secondary rainbows and mainly dominated by external reflection [10]. The rainbow angle is determined from the extremum of the angular deviation between the incident and emergent rays after internal reflections as the rainbow angle is the supplementary angle; $180^{\circ}$ - extremum scattering angle [4]. For rainbows observed in the sky, it is the angle of the elevation relative to the sun-observer line. For water droplets, the rainbow angle of the primary rainbow and secondary rainbows are about $42^{\circ}$ and $51^{\circ}$, respectively. The ray of the minimum deviation for $p=2$ is called the rainbow ray or Descartes ray and its angular position is denoted as the geometrical rainbow angle $\left(\theta_{\mathrm{rg}}\right)$. After the exit of the internally reflected rays $(p \geq 2)$ from a droplet, interference between rays of the same order (same $p$ ) and of different orders occurs. Thus, destructive and constructive interferences occur due to the phase difference forming supernumerary arcs inside the rainbow pattern.

The principal theory used to model the rainbow signal is the Airy theory that takes into account the diffraction process by using the Huygens' principle on the propagation of a wave front. For the primary rainbow, the Airy theory is able to predict the rainbow shape and to provide the gradual fading of rainbow within Alexander's dark band, taking into account the finite droplet size. However, the Airy theory is a scalar theory and all the effects relating to the vector aspect of the light were not taken into account. The predicted rainbow pattern from the Airy theory is accurate only for the angular position which is close to the Descartes minimum scattering angle or rainbow angle (the more distance from rainbow angle, the less accurate of scattered light description).

Later, Lorenz-Mie theory was able to provide an accurate description of the scattered light intensity distribution including all the types of "light rays". In 1890, Lorenz [12] introduced a theory of light scattering by a transparent sphere in the framework of Ether theory. Mie rediscovered the same result in the framework of Maxwell's electromagnetic theory [13]. Mie derived a solution for the scattering of an incident electromagnetic plane wave by a homogeneous spherical particle by solving Maxwell's equations. This solution consists of infinite series of terms where all sources of interaction are mixed together. As Lorenz and Mie obtained the same result, it can be called Lorenz-Mie theory. After that, Debye (1909) also introduced a solution consisting of an infinite series of terms but each source of interaction can be independently quantified [14]. The simulation of the light scattered by a spherical particle around the rainbow angle from Lorenz-Mie's and Debye's theory is very accurate but time consuming [15]. Finally, Nussenzveig developed the complex angular momentum method and applied it to the Debye expansion [16]. He introduced approximations depending on the angular region under study, the droplet refractive index and the type of ray of interest.

Considering results presented in Figure 2, there is not only the interference between internally reflected rays $(p=2)$ that produces the rainbow pattern. Interference between externally reflected light rays $(p=0)$ and the light rays that have undergone multiple internal reflections creates high-frequency oscillations on the supernumerary arcs (Overlay on the Airy fringes). This phenomenon is called the ripple structure. All the features of the ripple structure can be simulated with the Lorenz-Mie or Debye theories. An example of rainbow pattern according to the LorenzMie theory is shown in Figure 3. These different theoretical tools will be used in this study to process the experimental rainbows to determine the size and the refractive index of droplets. 


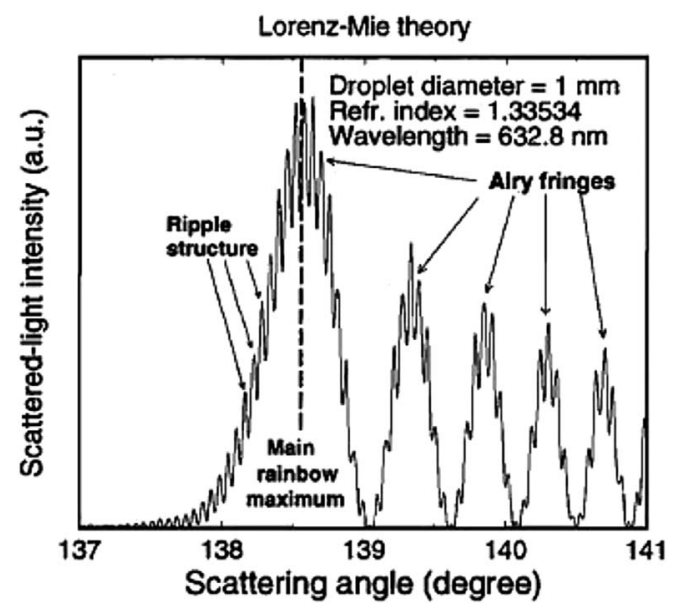

Fig. 3. Simulated monochromatic rainbow according to the Lorenz-Mie theory, Debye theory ( $p=0$ and 2), and Nussenzveig theory for a $1 \mathrm{~mm}$ droplet.

\section{Experimental setup}

This section describes the high-pressure optical test facility, the droplet stream generator as well as the optical measurement technique developed in the current study.

\subsection{High-pressure facility}

\subsubsection{Optical vessel}

An optical high-pressure test chamber was designed to produce the conditions of evaporation of fuel droplets in extreme thermodynamic conditions. For that, a monodispersed droplet stream injector (FMP-Technology) was inserted inside an optical high-pressure chamber in which the gas pressure can be monitored up to 50 bar. For a pressure above the critical pressure, the passage from sub- to supercritical conditions, when ethane is used, is controlled by changing the temperature of the gas from room temperature up to $35^{\circ} \mathrm{C}$. The main housing chamber, constructed in a stainless steel has an inner dimension surface of $45 \times 45 \mathrm{~mm}^{2}$ and a height of $168 \mathrm{~mm}$, the outer dimension are a width of $92 \mathrm{~mm}$ and a high of $168 \mathrm{~mm}$. It is equipped with four large UV quartz optical windows tailored to probe the droplet stream with optical diagnostics. Two windows of $40 \mathrm{~mm}$ wide and $150 \mathrm{~mm}$ height with a thickness of $20 \mathrm{~mm}$ are installed opposite to each other. The other sides are equipped with windows of $20 \mathrm{~mm}$ wide and $150 \mathrm{~mm}$ height with a thickness of $20 \mathrm{~mm}$. At the bottom part of the optical chamber, a dumping tank is used to store liquid during the experiment. This one is connected to the draining tube used to purge liquid at the end of the experiment. A schematic diagram of the high-pressure chamber is shown in Figure 4. For the control of temperature inside the test chamber, eight vertical electrical heat cartridges were positioned around its external surface. One type K thermocouple is installed into the test chamber wall to monitor and to control the wall temperature $\left(T_{\text {wall }}\right)$. To measure the gas temperature and ensure temperature uniformity

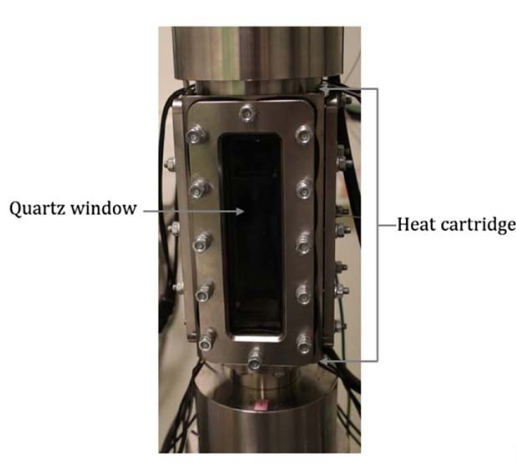

(a)

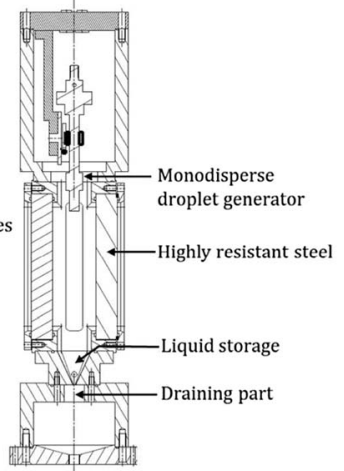

(b)
Fig. 4. High-pressure chamber: (a) front view image of the main housing, (b) design of the high-pressure test chamber.

throughout the pressure vessel $\left(T_{\text {gas,inside }}\right)$, three type $\mathrm{K}$ thermocouples are placed at different positions along the height of the visualisation module. The gas pressure $\left(P_{\text {gas }}\right)$ inside the pressure cell and the liquid pressure $\left(P_{\text {liquid }}\right)$ inside the injector were measured by a unique pressure transducer. The difference between the gas pressure and the liquid pressure $(\Delta P)$ was then obtained. Both temperature and pressure were monitored continuously during experiments in order to obtain reproducible experiments during time. All electronics connections between the PC computer, pressure transducer and temperature transducers were designed to assure a remote control. Therefore, the heating system, temperature measurement, pressure measurement, syringe pump and flowmeters can be remote controlled by a PC computer.

\subsubsection{Liquid supply system}

The liquid supply system is a key component for liquid fuel injection. The schematic diagram of the system is shown in Figure 5. Rigid stainless steel tubes (6 $\mathrm{mm}$ diameter) were used to connect together the syringe pump, the liquid supply and the monodispersed droplet stream generator. The syringe pump (Nexus 6000 high-pressure precision syringe pump) was used to gradually administer small amounts of liquid to the droplet stream injector. A $20 \mathrm{~mL}$ stainless steel syringe (19 mm diameter) was specifically selected to perform experiments under high-pressure conditions (pressure up to 50 bar). For the control of the liquid system, four ball valves were mounted on the system. The four valves are used as follows:

Filling the liquid supply system: The first valve installed between the liquid supply and the syringe pump was used to inject liquid inside the liquid supply. During this procedure, only the first valve was opened and the liquid was introduced into the syringe.

Filling the liquid injection system: The second valve was used to inject liquid contained into the syringe inside the high-pressure test chamber. During the feeding of liquid into the injection system, the first valve was closed. After draining the syringe, the second valve was closed and then the first valve was opened to refill the syringe again. This 


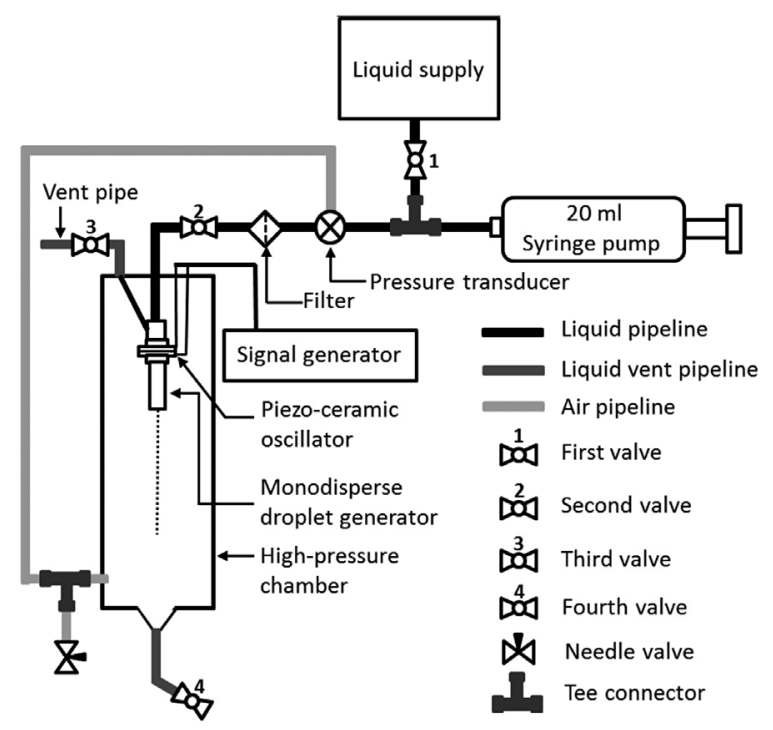

Fig. 5. Schematic diagram of the liquid injection supply.

sequence of procedure was then repeated several times to fully fill the liquid supply of the injector. However, air bubbles can subsist inside the liquid tubes.

Suppression of air bubbles: To eliminate air bubbles inside the liquid supply, a third valve was used. This valve was installed outside the test chamber on the purging pipe that is connected inside the test chamber to the injector. To drain air bubbles from the liquid supply system, the second and third valves were opened and the first valve was closed while liquid was injected. In this procedure, liquid and air bubbles escape through the vent pipeline to outside the test chamber. Once the liquid was free of air bubbles, the third valve is closed. According to this process, liquid fuel can be finally injected from the micro-hole as a fine liquid jet with a constant direction.

Draining of the used liquid: Liquid fuel was recovered inside the dumping tank located at the bottom part of the chamber. The dumping tank was connected to a stainless steel tube via a fourth valve. When opened, evacuation of the liquid fuel from the test chamber was performed. Therefore, the dump liquid fuel during the steps 1-3 was drained before starting the experiments in high-pressure conditions.

Control of liquid during experiment: To perform experiments in high-pressure conditions, only the second valve was opened. This procedure establishes a stream of liquid droplets into the high-pressure test chamber. Once the liquid fuel in the syringe was completely used during experiments, a refill procedure of the liquid supply with liquid was repeated to perform a new experiment.

Refilling of liquid during experiment: To refill liquid under high pressure condition, the second valve is firstly closed to prevent gas under high-pressure to flow out from the chamber and then the first valve was opened to refill the liquid supply. After that, the first valve was closed and the second valve was opened again to perform experiment. Due to the limitation of the volume of the syringe pump (20 mL), the liquid reservoir must be refilled several times during experiments. Although the procedure of running is complex in terms of its implementation, this one does not cause drawbacks during experiments in high-pressure conditions.

Draining liquid from the test chamber: At the end of the experiment, the liquid is drained by opening the fourth valve located outside the test chamber.

\subsubsection{Generation of monodispersed droplets}

In continuous jet technology, the monodispersed droplet generator relies upon the principle of applying a constant periodic excitation to a laminar liquid jet, which causes surface waves to form and grow as the jet slows down. Breakup into a single droplet per surface wave period thus occurs. Liquid injection is then a key process for generating a line of monodispersed droplets that have rigorously spherical shapes and stable falling rates. Controlling this process is obtained by properly choosing the generator frequency $\left(f_{\mathrm{G}}\right)$ and the volume flow rate $(V)$. The generator frequency is obtained by adapting the signal produced by a pulse generator for driving the piezoelectric transducer while the volumetric flowrate of liquid fuel is adapted by the speed of displacement of the piston integrated into the syringe pump. To excite the piezo-ceramic transducer, a voltage amplitude of 15 volts is fixed. The frequency generator is connected to the piezo-ceramic vibrating element of the injector as seen in Figure 5. The syringe pump (Nexus 6000 high-pressure precision syringe pump) is connected to the droplet generator by using the liquid tube supply described previously. With this system, the syringe pump is able to control the liquid flowrate in a large dynamic domain even in high-pressure conditions. In the current study, the volume flowrate ranges between 0.5 and $5 \mathrm{~mL} / \mathrm{min}$. Furthermore, a nozzle plate with a $100 \mu \mathrm{m}$ pinhole diameter $(D)$ was inserted inside the injector to produce monodispersed droplets. Conditions able to produce monodispersed droplets at atmospheric pressure were determined from calculations based on the theory on Rayleigh breakup of laminar fluid jets: it is noted that a liquid jet decays into droplets as stable criteria when the wavelength of deformation on the jet surface is larger than the circumference of the jet [17]. Based on theoretical calculations, the excitation frequency $\left(f_{\mathrm{G}}\right)$ corresponding to different volume flow rates $(V)$ able to produce monodispersed droplets with a pinhole diameter $(D)$ of $100 \mu \mathrm{m}$ are presented in Table 1. For experiments at atmospheric pressure, the volume flow rate was firstly set by fixing a flow rate with the syringe pump and then by adjusting the vibration frequency of the piezoceramic. Adjustment of the vibration frequency is optimized when the observed rainbow signal delivered by the spherical monodispersed droplets presents a perfect stable ripple structure. Such ripple structure will not happen when droplets size are not monodispersed or the shape of the droplet is not spherical or both.

Figure 6 shows an example of an image of rainbow light distribution with a detailed ripple structure. From an experimental point of view, the excitation frequency and the volumetric flowrate required to produce stable rainbow signals with ripple structures correspond with the values calculated from theoretical considerations. Accordingly, 


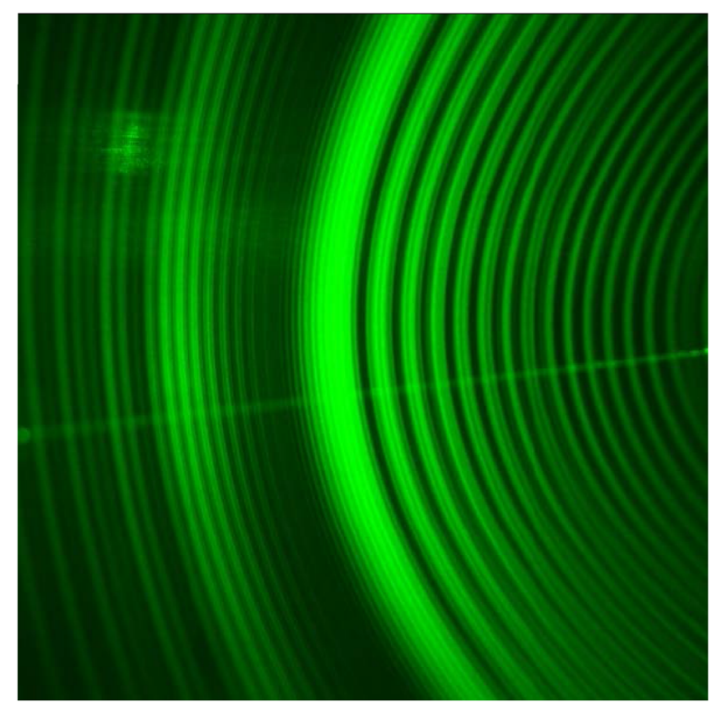

Fig. 6. Rainbow signal generated from a $50 \mu \mathrm{m}$ pinhole by setting a frequency of $25.091 \mathrm{kHz}$ frequency and $0.6 \mathrm{~mL} / \mathrm{min}$ of volume flowrate at the atmospheric pressure.

the volumetric flowrate and the range of corresponding frequencies from Table 1 are used to generate reproducible monodispersed droplets during experiments. For instance, for a pinhole size of $100 \mu \mathrm{m}$ and a volume flowrate of $2.2 \mathrm{~mL} / \mathrm{min}$, the excitation frequency within the range of $4.458-13.374 \mathrm{kHz}$ is required to produce monodispersed droplets. Accordingly, the expected droplet diameter $(d)$ produced from a $100 \mu \mathrm{m}$ pinhole is between 173 and $250 \mu \mathrm{m}$.

\subsubsection{Pressure control}

This section describes the procedure to control the pressure inside the test chamber. Synthetic air was used to pressurize the test chamber. The connection between the pressurization system and liquid supply system is shown in Figure 7. A pressure regulator was initially used to control the maximum input pressure of air stored in a compressed air bottle. The input air flowrate used to pressurize air inside the test chamber was then controlled by a mass flowmeter. With this system, the minimum and maximum flowrates that are used to pressurize air in the test chamber are ranging between 0.005 and $0.500 \mathrm{~g} / \mathrm{s}$, respectively. After ending experiments under high-pressure conditions, air contained

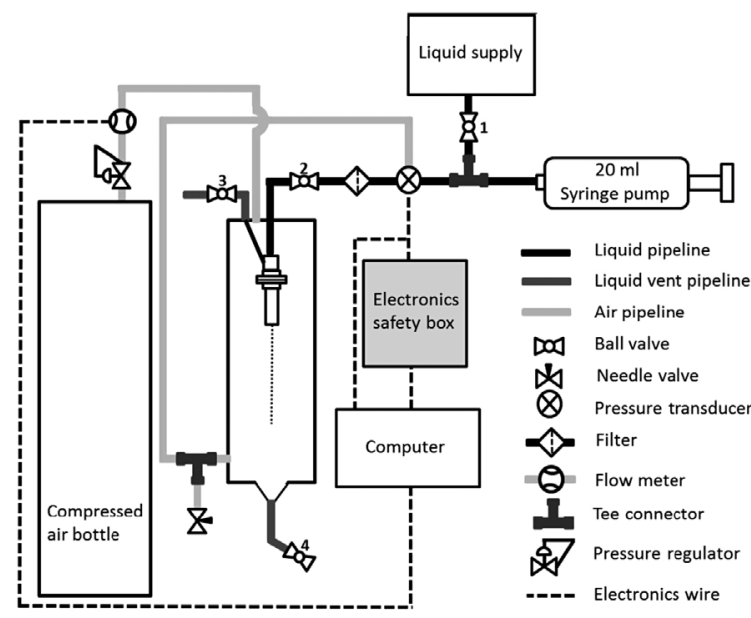

Fig. 7. Schematic diagram of the air pressurization system with the associated connection to the liquid injection system.

inside the test chamber was vented to decrease the pressure by using a needle valve. Moreover, this needle valve has been used during experiments to precisely control the pressure during experiments.

\subsubsection{Temperature control}

The test chamber is heated with eight vertical heat cartridges inserted around the external surface of the walls of the test chamber. A series of type-K thermocouples inserted at various positions inside the test chamber is used to control the heating of the test chamber and also to measure the temperature of gas during experiments. Figure 8 shows the locations of the type-K thermocouples positioned inside the test chamber. First of all, the control of the wall temperature was initially performed by using a type-K thermocouple inserted $\left(T_{\text {wall }}\right)$ inside the wall. Second, three type-K thermocouples were inserted inside the test chamber for precisely controlling the gas temperature at different locations. One is inserted in the module in which the injector was installed. Two another type-K thermocouples were positioned downstream from the exit injector; one is used to measure the boundary gas temperature $\left(T_{\text {gas2,inside }}\right)$ at the position in which droplets are injected inside the test chamber while the second one allows the measurement of gas temperature $\left(T_{\text {gas } 3 \text {,inside }}\right)$ at the location in which rainbow measurements were performed. Temperature of air outside

Table 1. Range of excited frequency relating to the volumetric flowrates for producing monodispersed droplets with a $100 \mu \mathrm{m}$ pinhole at atmospheric pressure.

\begin{tabular}{lccc}
\hline$V(\mathrm{~mL} / \mathrm{min})$ & $u_{D}(\mathrm{~cm} / \mathrm{min})$ & Range of $f_{G}(\mathrm{kHz})$ & Range of $d(\mu \mathrm{m})$ \\
\hline 1.2 & 15279 & $2.432<f_{G}<7.295$ & $250>d>173$ \\
1.4 & 17825 & $2.837<f_{G}<8.511$ & $250>d>173$ \\
1.6 & 20372 & $3.242<f_{G}<9.727$ & $250>d>173$ \\
1.8 & 22918 & $3.648<f_{G}<10.943$ & $250>d>173$ \\
2.0 & 25465 & $4.053<f_{G}<12.159$ & $250>d>173$ \\
2.2 & 28011 & $4.458<f_{G}<13.374$ & $250>d>173$ \\
\hline
\end{tabular}




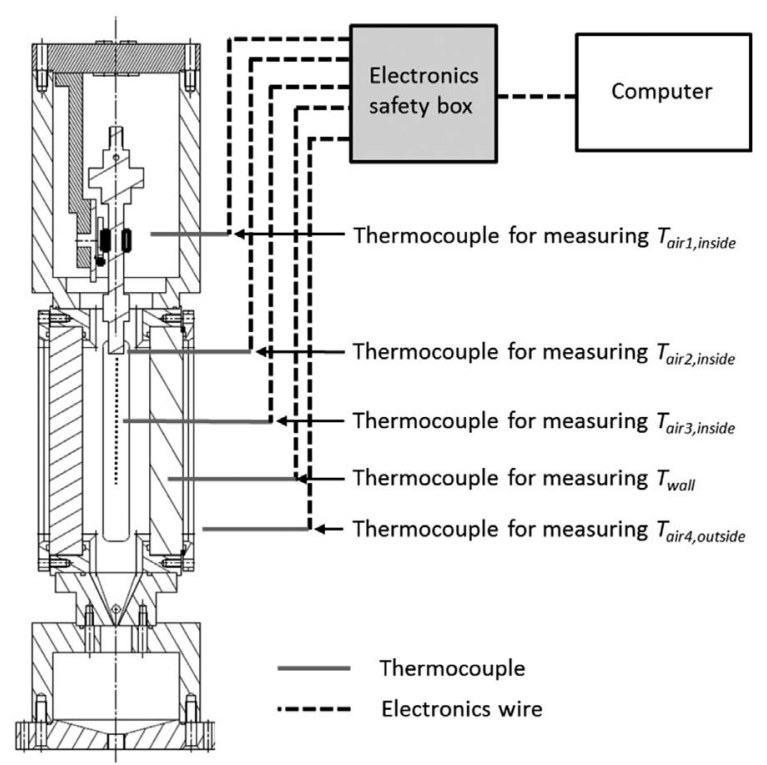

Fig. 8. The schematic diagram of temperature measurement.

the test chamber $\left(T_{\text {air,outside }}\right)$ was finally measured with a type-K thermocouple placed outside the test chamber.

\subsection{Rainbow technique and data processing}

As the angular rainbow position is sensitive to the refractive index value, it has been proposed to use it to measure the refractive index of the liquid droplet (see the pioneer work of Marston and Goosby [18] and Roth et al. [19, 20]). Basically, two strategies of measurements are proposed to measure the refractive index from the light scattered around the rainbow angle.

Standard configuration: In this configuration, the rainbow signal produced from the light scattered by a single droplet (or a series of droplets with exactly the same size and trajectory) is recorded and data processed. The interferences between internally reflected rays will induce a low frequency structure called Airy fringes. The interferences between internally and externally reflected rays will cause a high frequency structure named ripple structure superimposed on the Airy fringes. Using the Lorenz-Mie theory, which consists in resolving the Maxwell's equations, we can compute the rainbow scattered by a single droplet. Very accurate measurements of droplet size and refractive index can be obtained [21] but at the condition for which droplets must be perfectly spherical.

Global configuration: The global configuration has been introduced by Van Beeck et al. [22] to probe a number of droplets of different sizes (spray). The principle of the technique is to superimpose the rainbow patterns issued from enough droplets to be statistically representative of the spray so that the ripple structure totally disappears. The dependency of the rainbow to droplets temperature is then related to the fact that the index of refraction is a function of the droplet temperature. Data processing of the global

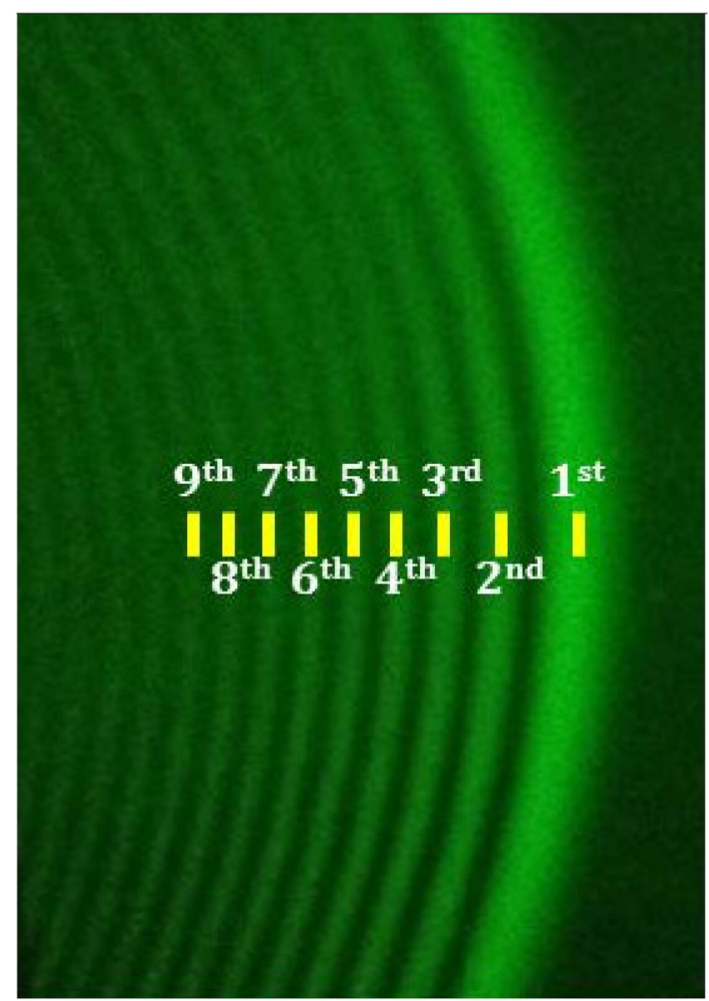

Fig. 9. Measurement of bright pattern positions.

rainbow pattern permits to extract a size distribution and an average refractive index. This approach is robust and can be applied with non-spherical droplets [23, 24]. This configuration has been successfully applied to measure moving droplet refractive index in different physical processes: combustion [25], $\mathrm{CO}_{2}$ capture [26], icing wind tunnel [27], to give few examples. Nevertheless, all these measurements have only been carried out at atmospheric pressure.

In the particular case of droplets injection moving in high-pressure conditions, the production of a line of perfectly spherical droplets is complex to reach, that leads to the record of a rainbow where only the "Airy" bows are visible (see Figs. 9 and 13b). Consequences of this result imply that the classical standard and the global rainbow approaches can not be directly applied. Accordingly, the following data processing has been applied. The peak positions of supernumerary bows were estimated and used to determine the mean droplet size and refractive index. To do this, the angular peak positions of supernumerary bows were compared to those obtained from the simulations based on Debye's theory $(p=2)$. Consequently, the mean size and the refractive index of nearly monodispersed droplets are extracted from the best fit peak positions obtained from the computed rainbow signals. As an example of data processing, Figure 9 shows an experimental monochromatic rainbow signal (laser wavelength: $0.532 \mu \mathrm{m}$ ) as well as the peak intensity positions of the bright patterns. Figure 10 presents the peak positions of supernumerary bows obtained from the measurement on water droplets under a pressure of 15.26 bar as the green vertical lines and its best 


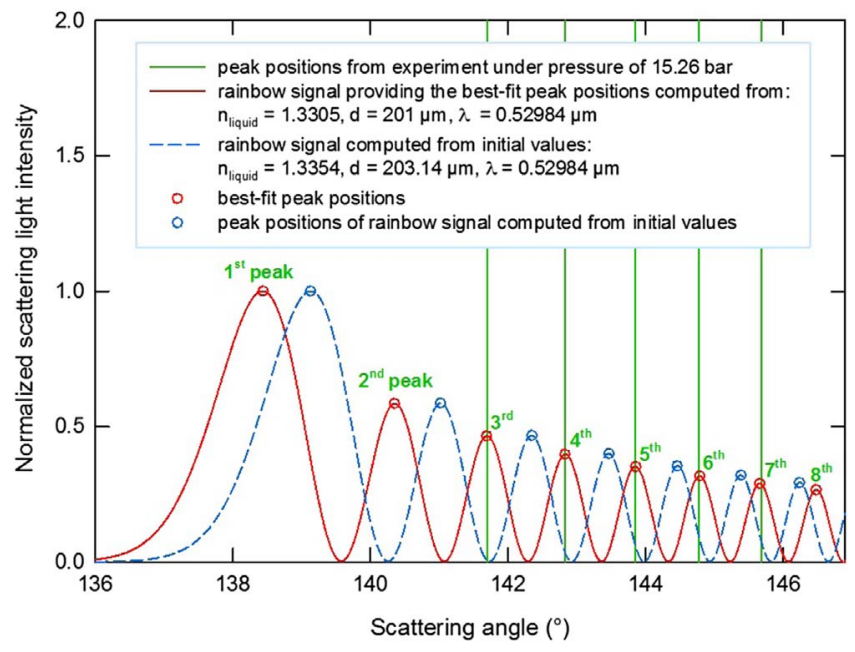

Fig. 10. Fitting of peak positions of these simulated rainbow signals to the maximum intensity positions of bright patterns of water droplets. Air pressure is equal to 15.26 bar.

fit peak positions obtained from the rainbow pattern simulations based on the Debye's theory $(p=2)$ as the red curve. The rainbow computed from the initial data is plotted as a dashed blue line. It is noted that this procedure allows measurements of the size and refractive index, including cases where only a part of the rainbow signal is recorded as shown in Figure 10.

\subsubsection{Refractive index of air}

The refractive index of air is another key parameter used to calculate the scattering angle. Furthermore, this one is function of wavelength, temperature and pressure. During experiments, scattered light was observed under various thermodynamic conditions. Therefore, refractive index of air was computed from the experimental conditions. In the current study, the relationship of the refractive index of air with temperature, pressure and wavelength was calculated by the Edlén correlations (Edlén [28]):

$$
\begin{aligned}
\left(n_{\mathrm{air}, P}-1\right) & =\frac{P_{\mathrm{AIR}}\left(n_{\mathrm{air}, 1}-1\right)_{s}}{96095.4} \\
& \times \frac{\left[1+P_{\mathrm{AIR}}\left(61.3-T_{\mathrm{air}, \text { in }}\right) \times 10^{-10}\right.}{\left(1+0.00366 T_{\mathrm{air}, \text { in }}\right)},
\end{aligned}
$$

where $n_{\mathrm{air}, P}$ is the refractive index of air in test chamber at $T_{\text {air;in }}\left({ }^{\circ} \mathrm{C}\right)$ and pressure $P_{\mathrm{AIR}}$ in $\mathrm{Pa}$ and $\left(n_{\mathrm{air}, 1}-1\right)_{s}$ is given by:

$$
\left(n_{\text {air }, 1}-1\right) \times 10^{-8}=8342.13+\frac{2406030}{\left(130-\sigma^{2}\right)}+\frac{15997}{\left(38.9-\sigma^{2}\right)},
$$

$\sigma=1=\lambda_{\text {vac }}$ where $\lambda_{\text {vac }}$ is the wavelength of the radiation in vacuum in $\mu \mathrm{m}$.

Equations (1) and (2) were used to calculate the refractive index of air as function of the pressure in the test chamber $\left(n_{\text {air }, P}\right)$ and the refractive index of air outside the test chamber at atmospheric pressure $\left(n_{\text {air }, 1}\right)$, respectively. Table 2 shows refractive index of air at various pressures calculated from Equation (1). The relationship between refractive index of air and pressure is presented in Figure 11. From Table 2, refractive index of air outside the test chamber $\left(n_{\text {air. }, 1}\right)$ at temperature of $20{ }^{\circ} \mathrm{C}$ is 1.00027 . Refractive index of air inside the test chamber $n_{\mathrm{air}, P}$ was calculated by using the pressure recorded during the experiment.

\section{Results}

This section demonstrates the potential to measure the refractive index (and so the temperature of droplets) with rainbow refractometry on moving droplets expanding in high-pressure environments. To the best of our knowledge, these results are unique in the literature. Therefore, the validation of this optical technique has been carried out firstly. Water and ethanol liquid fluids were selected for this experiment of validation as their refractive index was measured in the past in high-pressure optical cells filled with these liquids [3-7]. Accordingly, the refractive index of water and ethanol measured by the rainbow technique was then compared with the values published in the literature. Then, experiments were performed on ethane fuel which is considered as a good working fluid in extreme pressures because this molecule exhibits attractive thermodynamic critical conditions easily attainable in a laboratory experiment. To illustrate this point of view, Figure 12 synthetizes the pressure-temperature phase diagram for water, ethanol and ethane. The thermodynamic conditions in which rainbow measurements were performed for water and ethanol fuels are represented by grey triangles, these ones being far from the critical points of water and ethanol (blue star and yellow star, respectively). On the other hand, the operating condition for ethane droplets is represented as the black triangles that can be close to its critical point (green star).

\subsection{Water}

At atmospheric pressure, the production of a line of monodispersed droplets was achieved and stable rainbow signals with its ripple structure were observed. By contrast, the experimental rainbow signals recorded under high-pressure conditions were not stable and the ripple structure disappeared. Examples of rainbow signals observed under pressure of 20 bar are shown in Figure 13. All the recording include in that paper have been recorded with a probe volume of about $1 \mathrm{~mm}^{3}$. The laser beam is cylindrical with a diameter equal to about $1 \mathrm{~mm}$. The number of droplets creating the signal depends on the droplet generator frequency and the camera exposure time. Typically about 1000-2000 droplets create the rainbow signals processed in that paper. Figure 13a presents the rainbow signal observed from a nearly monodispersed spray produced at a flow rate $V$ of $2.2 \mathrm{~mL} / \mathrm{min}$ whereas Figure $13 \mathrm{~b}$ shows the rainbow signal observed from nearly monodispersed spray produced at $V$ of $1.6 \mathrm{~mL} / \mathrm{min}$. The rainbow signal 
Table 2. Refractive index of air with pressure.

\begin{tabular}{cccc}
\hline $\begin{array}{l}\text { Pressure } \\
\left(P_{\text {AIR }}\right)(\mathrm{Pa})\end{array}$ & $\begin{array}{c}\text { Pressure }\left(P_{\text {air }}\right) \\
(\text { bar })\end{array}$ & $\begin{array}{c}T_{\text {air }} \\
\left({ }^{\circ} \mathrm{C}\right)\end{array}$ & $\begin{array}{c}\text { Refractive index } \\
\text { of air }\left(n_{\text {air }, P}\right)\end{array}$ \\
\hline 100000 & 1 & 20 & 1.00027 \\
101325 & 1.01325 & 20 & 1.00027 \\
500000 & 5 & 20 & 1.00135 \\
1000000 & 10 & 20 & 1.00271 \\
1500000 & 15 & 20 & 1.00407 \\
2000000 & 20 & 20 & 1.00544 \\
2500000 & 25 & 20 & 1.00681 \\
3000000 & 30 & 20 & 1.00819 \\
3500000 & 35 & 20 & 1.00958 \\
4000000 & 40 & 20 & 1.01097 \\
4500000 & 45 & 20 & 1.01236 \\
5000000 & 50 & 20 & 1.01377 \\
\hline
\end{tabular}

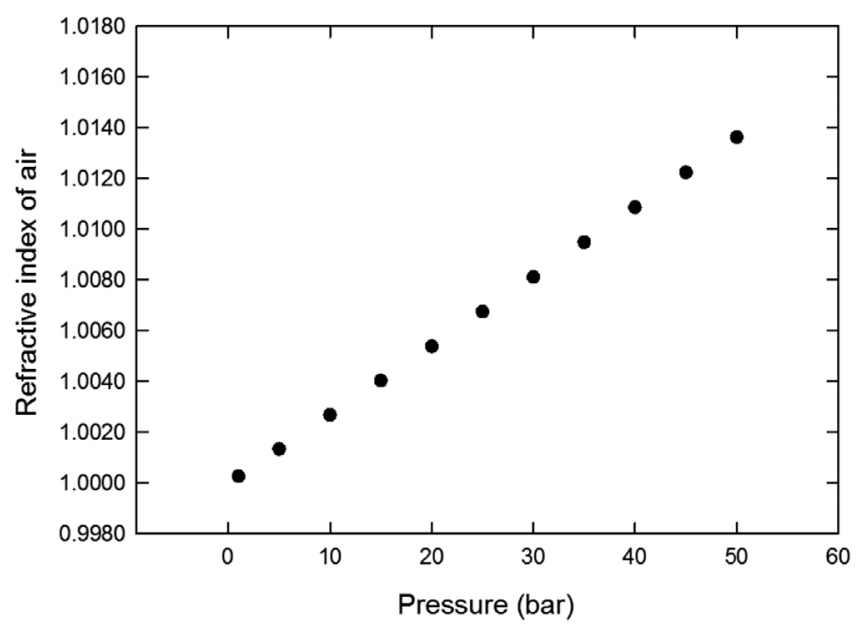

Fig. 11. Relationship between refractive index of air and pressure.

in Figure 13a is more blurry than in Figure 13b due to the existence of jet instabilities in the test chamber. The better signal produced at $V$ of $1.6 \mathrm{~mL} / \mathrm{min}$ was obtained after adjusting $V$.

When the pressure in the test chamber increases, the rainbow signal is shifted at smaller scattering angle. After determining the angular positions corresponding to each peak, results were fitted with simulated data to determine the relative refractive index $\left(n_{\text {water, } P}\right)$ and droplets size. Figure 14 displays the measured refractive index of water droplets versus pressure for three experiments (circle blue, red and green points with error bars). As observed in this figure, a good reproducibility of the experiments was achieved despite the complex operating conditions encountered in the test chamber. It is also noted that the relative refractive index of water decreases when the pressure in the test chamber increases. On Figure 14 are also plotted the absolute refractive index value of water measured by Schiebener et al. [29] as yellow triangles. Results of

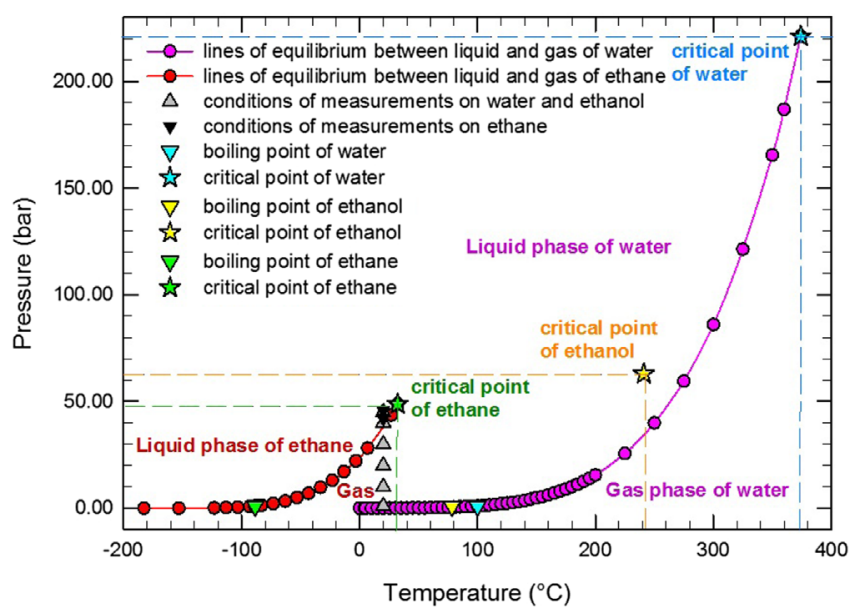

Fig. 12. Equilibrium curve between liquid and gas for water and ethane. The thermodynamic conditions of measurements on water, ethanol and ethane droplets are also indicated.

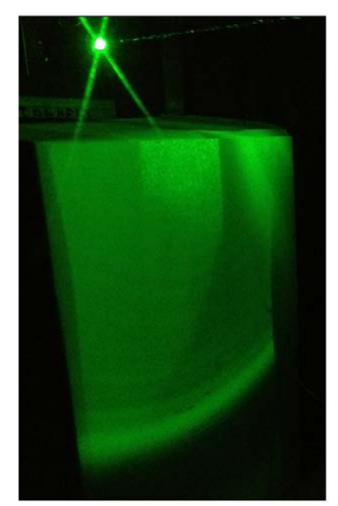

(a)

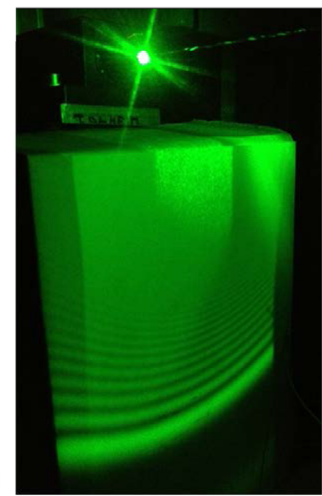

(b)
Fig. 13. Rainbow signals observed under pressure of 20 bar (a) rainbow signal produced at $V$ of $2.2 \mathrm{~mL} / \mathrm{min}$ (b) rainbow signal after adjusting $V$ to $1.6 \mathrm{~mL} / \mathrm{min}$.

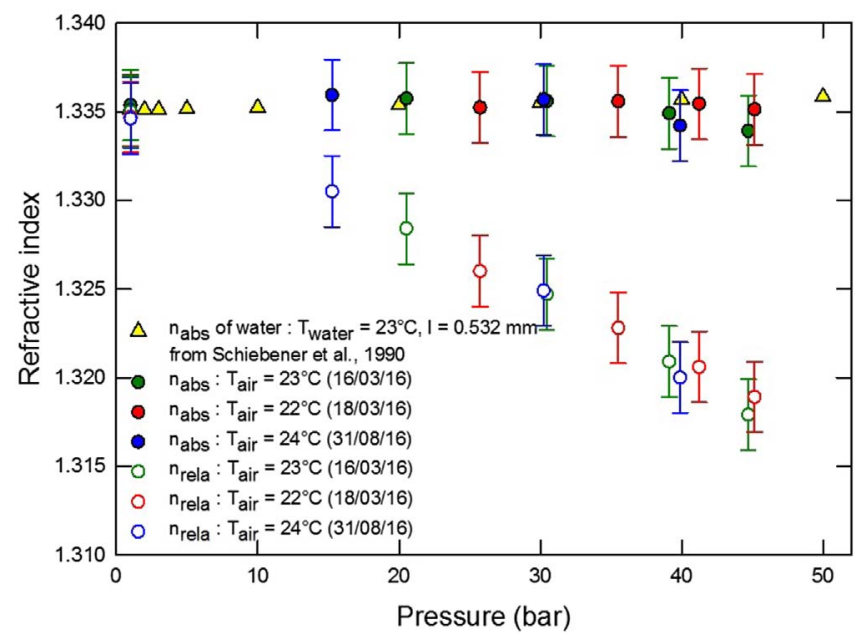

Fig. 14. Pressure dependence of the relative and absolute refractive index of water droplets. 
Table 3. Water measurement data, calculated data and results of rainbow signal fitting process.

\begin{tabular}{lccccccc}
\hline$P_{\text {inside }}(\mathrm{bar})$ & $T_{\text {air,inside }}\left({ }^{\circ} \mathrm{C}\right)$ & $T_{\text {air,outside }}\left({ }^{\circ} \mathrm{C}\right)$ & $d_{\text {theoretical }}(\mu \mathrm{m})$ & $d_{\text {fitting }}(\mu \mathrm{m})$ & $n_{\text {air }, P}$ & $n_{\text {rela }}$ & $n_{\text {abs }}$ \\
\hline 1.01 & 23.90 & 24.40 & 167.40 & 165 & 1.0002698 & 1.3346 & 1.3350 \\
15.26 & 24.10 & 24.80 & 203.14 & 201 & 1.0040826 & 1.3305 & 1.3359 \\
30.24 & 24.20 & 24.90 & 183.24 & 179 & 1.0081322 & 1.3249 & 1.3357 \\
39.90 & 24.40 & 25.10 & 181.89 & 179 & 1.0107599 & 1.3200 & 1.3342 \\
\hline
\end{tabular}

Schiebener et al. display a small increase of the water refractive index with pressure. For experiments under highpressure conditions, the density of air inside the test chamber changes due to the pressure as well as its refractive index $\left(n_{\mathrm{air}, P}\right)$. From this reason, the refractive index of air $\left(n_{\text {air }, P}\right)$ must be included into the data processing of the Rainbow signals for the true calculation of the absolute refractive index of droplets. For water, thermodynamic conditions in which droplets are injected are far from its critical point (critical pressure of 221 bar and critical temperature of $374^{\circ} \mathrm{C}$ ). Therefore, evaporation of liquid water droplets is a weak process in these experiments and only pure air surrounding water droplets can be considered. Absolute refractive index was then determined from the relative refractive index and absolute refractive index of air inside the test chamber. Results are shown in Table 3 . This table gives the pressure of air inside the test chamber $\left(P_{\text {inside }}\right)$, the temperature of air inside the test chamber $\left(T_{\text {air.inside }}\right)$, the temperature of air outside the test chamber $\left(T_{\text {air.outside }}\right)$, the droplet size calculated from volumetric flow rate and excitation frequency $\left(d_{\text {theoretical }}\right)$, the droplet size obtained from data processing $\left(d_{\text {fitting }}\right.$, the refractive index of air due to pressure $\left(n_{\text {air }, P}\right)$, the relative refractive index $\left(n_{\text {liquid }, P}\right)$ and the absolute refractive index determined from measurements $\left(n_{\text {liquid }, P}\right)$ performed for a series of measurements recorded during the same experiment. It is then found that the average diameter of droplets obtained from the data fitting analysis is in good agreement with those calculated from the volumetric flowrates and excitation frequency in the range of $\pm 5 \mu \mathrm{m}$.

Figure 14 shows the relative and absolute refractive indices of water at different pressures determined from the fitting with an uncertainty of 0.002 , and absolute refractive indices from Schiebener et al. [29]. As observed in this figure, the experimental behaviour of water absolute refractive index with pressure carried out on free falling droplets are in well agreement with the results of Schiebener.

\subsection{Ethanol}

In order to confirm the reliability of the experimental methodology used to process the rainbow patterns recorded in high-pressure conditions, measurements of refractive index of free-falling ethanol droplets under pressure up to 30 bar were carried out. In this case, the gas surrounding droplets is not only pure air but a mixture of air with evaporated ethanol. The behaviour of the relative refractive index of ethanol droplets with pressure is compared with the behaviour recorded for water (see Fig. 15). The relative refractive index of both liquid water and ethanol decreased when the pressure increases. As seen in Figure 15, the

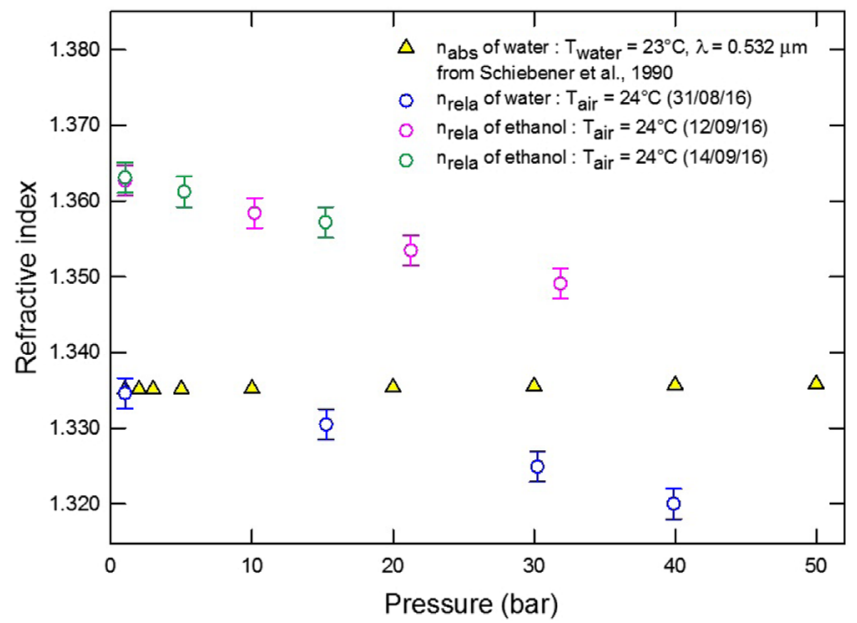

Fig. 15. Pressure dependence of relative refractive index of liquid ethanol and water droplets.

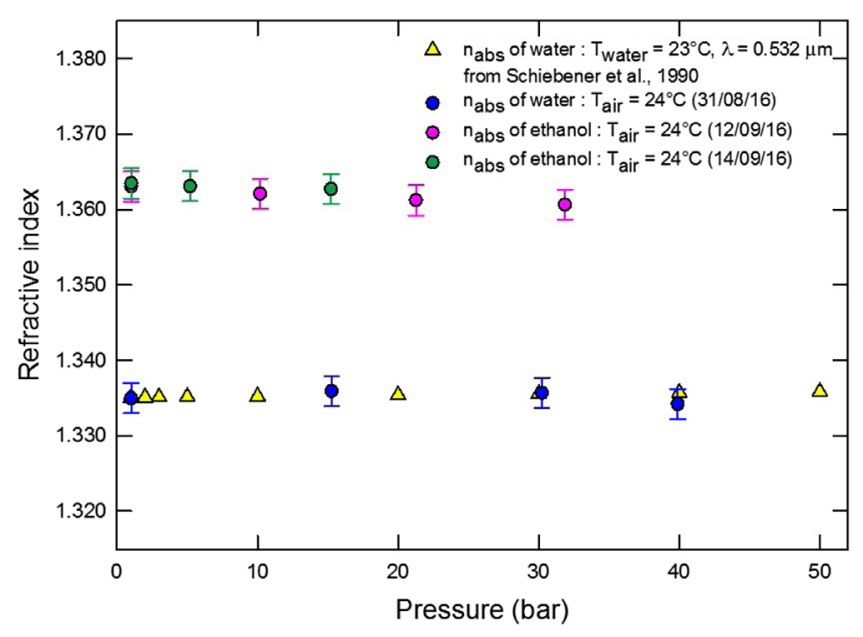

Fig. 16. Pressure dependence of the absolute refractive index of liquid ethanol and water droplets.

decrease of the relative refractive indices of ethanol and water are quite similar. A correction of the effect of the refractive index of air on the experimental results allows to determine the absolute refractive index of ethanol. The evolution of the absolute refractive index of ethanol droplets with pressure presents a small decrease similarly to the one observed on water droplets (Fig. 16).

Table 4 shows the measurement data, simulation data and results of the rainbow signal fitting process from the 
Table 4. Ethanol measurement data, calculated data and results of rainbow signal fitting process.

\begin{tabular}{lccccccc}
\hline$P_{\text {inside }}($ bar $)$ & $T_{\text {air,inside }}\left({ }^{\circ} \mathrm{C}\right)$ & $T_{\text {air,outside }}\left({ }^{\circ} \mathrm{C}\right)$ & $d_{\text {theoretical }}(\mu \mathrm{m})$ & $d_{\text {fitting }}(\mu \mathrm{m})$ & $n_{\text {air }, P}$ & $n_{\text {rela }}$ & $n_{\text {abs }}$ \\
\hline 1.01 & 23.50 & 23.60 & 186.08 & 186 & 1.0002702 & 1.3627 & 1.3631 \\
10.19 & 24.00 & 24.10 & 187.40 & 177 & 1.0027220 & 1.3584 & 1.3621 \\
21.27 & 24.30 & 24.40 & 235.66 & 212 & 1.0056991 & 1.3535 & 1.3612 \\
31.87 & 24.60 & 24.60 & 216.569 & 203 & 1.0085630 & 1.3491 & 1.3607 \\
\hline
\end{tabular}

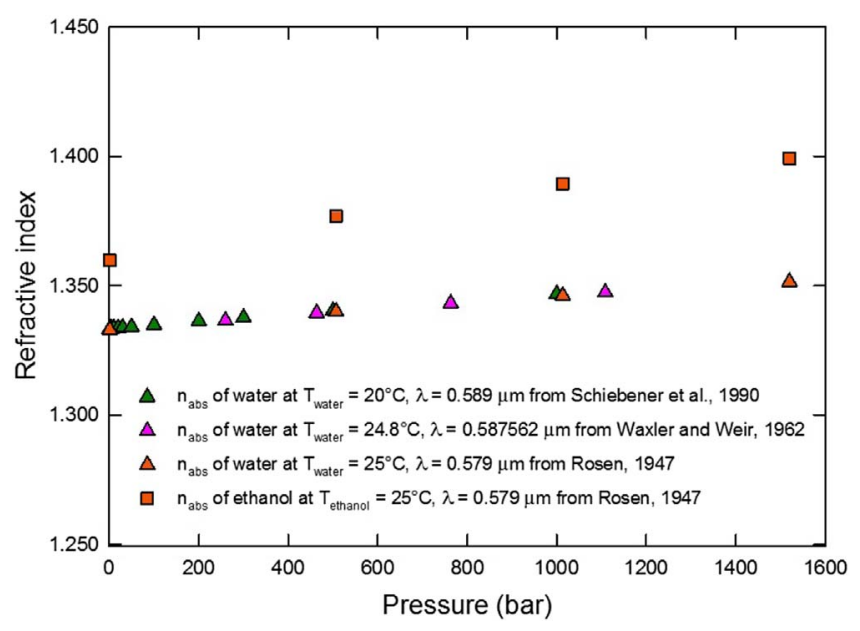

Fig. 17. Pressure dependence of the absolute refractive index of liquid ethanol and water from the literature.

measurements recorded on ethanol droplets. It is noticed that the average diameter from the fitting analysis was smaller than those calculated from the volumetric flow rate and excitation frequency by $10-20 \mu \mathrm{m}$. Unlike liquid, no evolution of ethanol refractive index reference data with pressure in the range in which measurements were performed (1-30 bar) was found in literature. However, reference data on the relationship between absolute refractive index and pressure at very high pressure (100-1000 bar) or liquid ethanol and water are accessible (Fig. 17). This figure shows that the absolute refractive index of liquid ethanol and water increase with pressure while Figure 18 displays the water refractive index dependence with pressure and temperature $\left(23\right.$ and $\left.50{ }^{\circ} \mathrm{C}\right)$.

\subsection{Ethane}

To perform experiments in a situation in which thermodynamic conditions of injection of liquid fuels are close to transcritical conditions, ethane was selected as a good probe molecule in regards to its attractive critical properties which can be easily obtained in laboratory experiments.

To produce ethane droplets, ethane vapor contained in the stored bottle is first pressurized at a pressure higher than 40 bar (critical pressure) to be converted into liquid. Figure 19 shows the pressure-temperature diagram in which the working zone investigated in the current study is referenced by the red circle while the blue triangle denotes the thermodynamic state of ethane vapor inside the storage bottle. The red and green triangles represent the

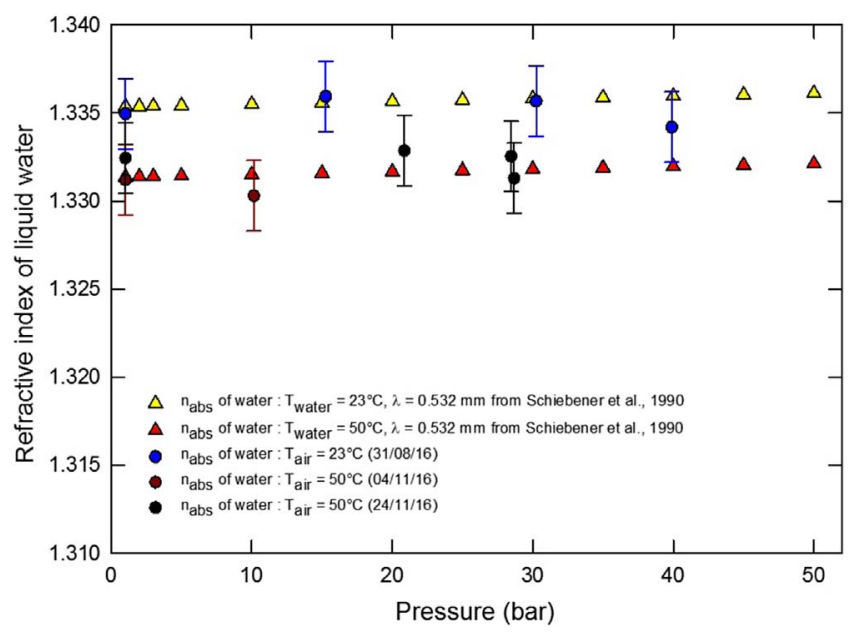

Fig. 18. Pressure and temperature dependence of absolute refractive index of water droplets.

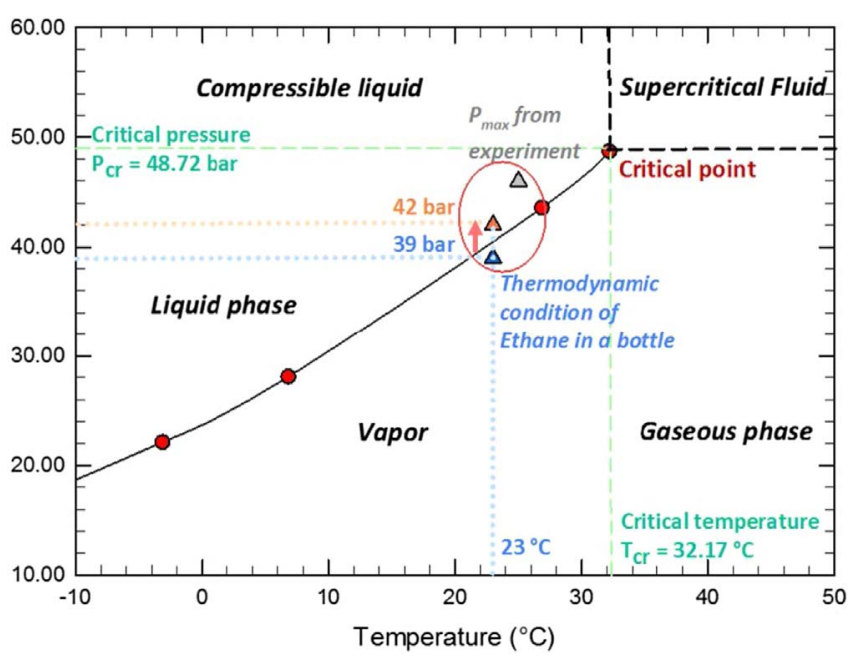

Fig. 19. Status of ethane for the measurements in the currently study on pressure-temperature diagram.

experimental points in which liquid ethane droplets were optically probed.

To achieve these conditions, the fluid supply system that was initially designed for water and ethanol was modified to guarantee a full conversion of ethane vapor into liquid before injection inside the high-pressure cell. As the volume of liquid of the supply system has small dimensions, 
Table 5. Summary of results obtained on the processing of rainbow signals of ethane droplets.

\begin{tabular}{lccccccc}
\hline Injection & $P_{\text {inside }}$ & $T_{\text {inside }}$ & $d_{\text {fitting }}$ & $n_{\text {air }, P}$ & $n_{\text {rela }}$ & $n_{\text {gas }, P}$ & $n_{\text {abs }}$ \\
\hline 1st & 42.50 & 22.00 & 140 & 1.0115768 & 1.2404 & 1.0116 & 1.2548 \\
2nd & 42.44 & 22.20 & 124 & 1.0115514 & 1.2365 & 1.0148 & 1.2548 \\
3rd & 42.43 & 22.50 & 123 & 1.0115354 & 1.2324 & 1.0182 & 1.2548 \\
\hline
\end{tabular}

Table 6. Percentage of the estimated concentration of ethane vapor from rainbow measurements.

\begin{tabular}{lccccc}
\hline Experiment & Injection & $P_{\text {inside }}($ bar $)$ & $T_{\text {inside }}\left({ }^{\circ} \mathrm{C}\right)$ & \multicolumn{2}{c}{ Gas concentration $(\%$ by mole) } \\
\cline { 5 - 6 } & & & 22.20 & Ethane & Air \\
\hline First & 1st & 42.50 & 22.50 & 0 & 7.4 \\
& 2nd & 42.44 & 22.50 & 15.2 & 92.6 \\
& 3rd & 42.43 & & & 84.8 \\
Second & & 41.29 & 21.7 & 4.3 & 900 \\
& 1st & 41.26 & 21.6 & 9.5 & 90.5 \\
& 2nd & 41.13 & 21.6 & 10.3 & 89.7 \\
\hline
\end{tabular}

the duration of production of liquid droplets during one injection process is short, typically a few dozen of seconds. This constraint requires to elaborate a strategy of measurements ensuring a detailed data processing of the rainbow signals. Three successive jets of free-falling ethane droplets at an inlet pressure of 42 bar and temperature of $23^{\circ} \mathrm{C}$ were then carried out. For each injection of liquid ethane, a rainbow signal was acquired and data processed. The effect of the species composition of the surrounding gas around droplets on the rainbow measurements was studied and the performances to probe liquid hydrocarbon fuels injected in conditions close to the critical point were evaluated. For the first jet, ethane vapor was pressurized at pressure ranging between 39 bar and 42 bar to ensure the conversion of ethane vapor into liquid into the supply system. A first jet of ethane droplets was done and the record of a rainbow signal was performed. Same experiment was then repeated two times more. Unexpectedly, the rainbow signal measured during the second injection shifted down in angle compared to the one recorded during the first injection. Furthermore, the rainbow signal observed during the third injection shifted once again down from the rainbow pattern recorded during the second injection.

This effect is simply the result of the modification of the species composition of the gaseous mixture surrounding the droplets during the successive injections of liquid fuel. Indeed, the amount of ethane vapor into air was increased under the evaporation of liquid droplets in transcritical conditions. Inevitably, the shifting of the rainbow signal during the second injection of ethane droplets was derived from the species composition of the gaseous mixture which was composed of a mixture of air and an amount of ethane vapor produced from the evaporation of ethane liquid during the first injection. This new chemical composition modifies the refractive index of the gaseous fluid around droplets and consequently, changes the diffusion angle of the rainbow signal. As the third injection of ethane droplets was performed in a gaseous mixture which was not purged at the end of the second injection, the amount of ethane vapor inside the gaseous mixture was necessarily larger, leading to an amplification of the shifting of the rainbow signal.

To estimate the amount of ethane vapor mixed with air, a new procedure has been developed and validated. During the first injection of liquid ethane, the absolute refractive index of the free-falling ethane droplets was deduced from the data processing of the rainbow signal, assuming a negligible amount of ethane vapor into the gaseous mixture. This means that the gaseous mixture surrounding the droplets is only composed of air. The strategy of measurement of the refractive index of liquid ethane is then the one initially developed for the case of water droplets. This refractive index, equal to $1.2545 \pm 0.002$, is then used as the absolute refractive index value of liquid ethane droplets when the rainbow signals during the second and third injections are recorded. The relative refractive index of ethane droplets measured during the second and third injections of liquid ethane combined with the absolute refractive index allowed us to get the refractive index of the surrounding gas mixture during these experiments. Results are shown in Table 5. A detailed analysis of these results shows that the absolute refractive index of the gaseous mixture increases successively with the number of injections of liquid fuels, demonstrating that the species concentration of ethane vapor increases sequentially when liquid ethane droplets are successively injected. In parallel to these measurements, the average size of ethane droplets were also measured from the analysis of the rainbow signals and they are in the range of 120-150 $\mu \mathrm{m}$ (Tab. 5).

From the absolute refractive index of the gaseous mixture in the test chamber, the concentration of ethane vapor 


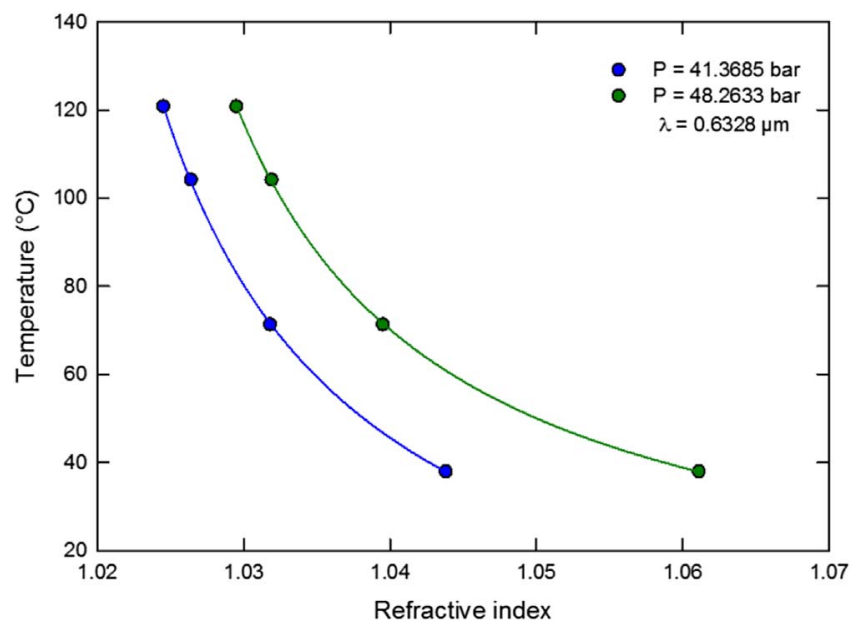

Fig. 20. Evolution of the refractive index of gaseous ethane with temperature for two pressure levels.

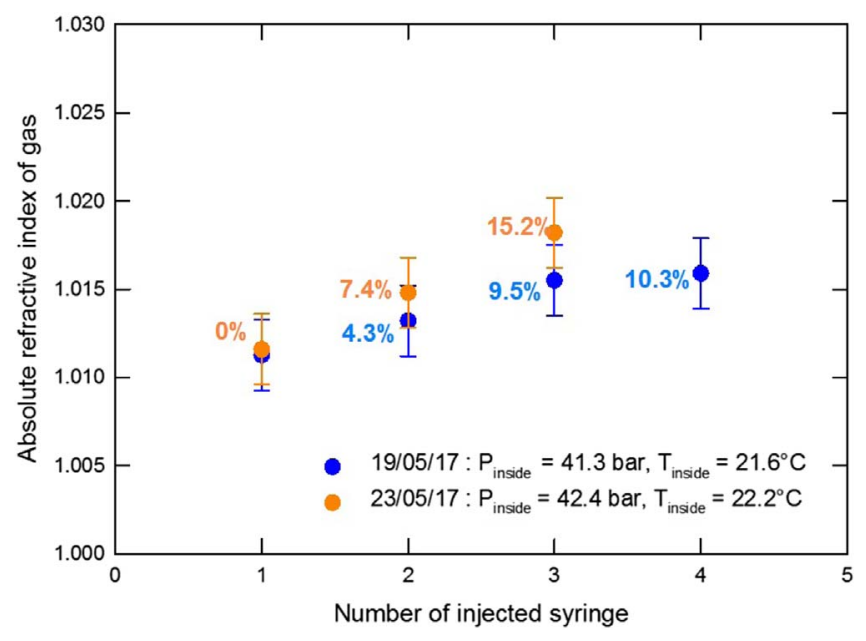

Fig. 21. Refractive index of the gaseous mixture measured into the test chamber versus the number of injection of liquid ethane. The percentages of ethane vapor concentration are also indicated.

in the test chamber for each measurement was estimated. Results of the concentration of ethane vapor for different injections are shown in Table 6. According to this procedure, the absolute refractive index of the gaseous fluid into the test chamber $\left(n_{\text {gas }, P}\right)$ and the relative refractive index of ethane droplet after the first injection of liquid ethane were determined from the absolute refractive index of ethane droplet $\left(n_{\text {ethane, } P}\right)$ determined after the first injection. Knowing the absolute refractive index of gas $\left(n_{\text {gas }, P}\right)$, the concentration of ethane vapor into the high-pressure chamber $(x)$ can be estimated from the following equation:

$$
n_{\text {gas }, P}=x \times n_{\text {ethane-vapor }, P}+(1-x) n_{\text {air }, P} .
$$

In this equation, the refractive index of air $n_{\mathrm{gas}, P}$ at pressure $P$ is calculated by equation (3) while the absolute refractive index of ethane vapor $\left(n_{\text {ethane-vapor }, P}\right)$ is deduced from data issued from the work of Besserer and Robinson [7]. In this study, relationships between the refractive index of ethane vapor and pressure and temperature are given. For instance, Figure 20 shows the evolution of the refractive index of ethane vapor with temperature for two values of pressure which have been investigated in the current study.

Data of ethane vapor concentration measured during the different fuel injections are resumed in Table 6 while Figure 21 displays the evolution of the absolute refractive index of the gaseous mixture versus the number of injections of liquid ethane. According to the results shown in this graph, the absolute refractive index of the gaseous mixture increases quasi-linearly with the number of injections of liquid ethane. For each point of measurement, the estimated percentage of ethane vapor in the gas mixture was indicated. During the first injection of ethane, the percentage of ethane vapor after this experiment performed at a pressure of 41 bar is about $7.4 \%$. After the second injection, the ethane vapor concentration increased to about $15.2 \%$, typically two times the quantity of ethane vapor measured after the first injection. For a second series of similar experiments performed at larger pressure, the percentage of ethane vapor after the two injections of liquid ethane at pressure of 42 bar are $4.3 \%$ and $9.5 \%$, respectively. As noted before, the concentration after the second injection is also twice the concentration of ethane vapor recorded after the first injection.

Furthermore, the ethane vapor concentration into the gaseous phase can be estimated from the conditions in which liquid ethane has been injected into the high-pressure chamber. First, a computed mass of liquid ethane can be estimated knowing the duration of the injection of liquid during the experiment. This one is then estimated to $1.15 \mathrm{~g}$ (i.e. 0.038 mole). The ethane vapor concentration produced during the three successive experiments are then calculated assuming a full evaporation of ethane during the injection process (see results in Tab. 7). As observed into this table, an ethane vapor concentration of about $5 \%$ into the gaseous mixture was then estimated after the first injection. This value was increased to about $10 \%$ after the second injection. A comparison of these values with those deduced from the rainbow refractometry measurements shown in Table 6 shows similar tendencies, namely a similar increase in proportion of the ethane vapor concentration with the number of injections of liquid ethane. Furthermore, a comparison with independent experiments shows similar trends on the impact of the ethane vapor concentration on the determination of the refractive index of liquid droplets in elevated pressure injection of fluids. As the determination of the ethane vapor concentration is only an estimated concentration for which the accuracy is only acceptable, this result highlights the requirement to put in place simultaneously complementary measurements of ethane vapor concentration with the rainbow measurements. A convenient way will be then to combine absorption measurements to measure the ethane vapor concentration with rainbow refractometry measurements to deduce the refractive index of liquid ethane droplets.

Finally, the relative and absolute refractive indices of ethane determined in the current study are presented 
Table 7. Estimated percentage of the concentration of ethane vapor in the gaseous mixture surrounding the ethane droplets injected in the high-pressure test chamber.

\begin{tabular}{lcccc}
\hline Experiment & Injection & $P_{\text {inside }}($ bar $)$ & $\begin{array}{c}T_{\text {inside }} \\
\left({ }^{\circ} \mathrm{C}\right)\end{array}$ & $\begin{array}{c}\text { Ethane concentration (\% by mole) } \\
\text { estimated for full evaporation }\end{array}$ \\
\hline First & 1st & 41.29 & 21.7 & 0 \\
& 2nd & 41.26 & 21.6 & 5.3 \\
& 3rd & 41.13 & 21.6 & 10.3 \\
\multirow{3}{*}{ Second } & 4 th & 41.29 & 21.6 & 15.1 \\
& & 42.50 & 22.20 & 0 \\
& 1st & 42.44 & 22.50 & 5.1 \\
& 2nd & 42.43 & 22.50 & 10.6 \\
\hline
\end{tabular}

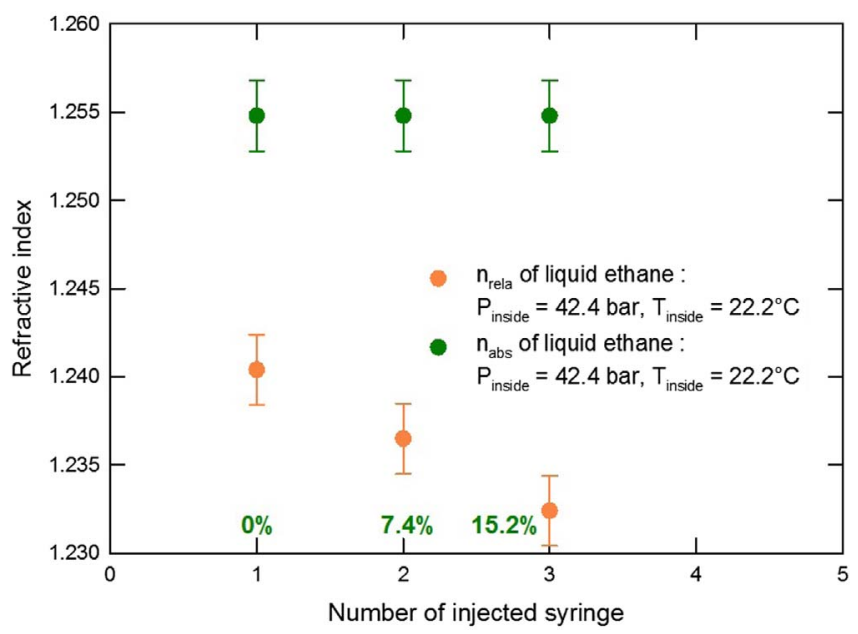

Fig. 22. Absolute and relative refractive index of liquid ethane droplets results number of times of injection of liquid ethane and the estimated percentage of ethane vapor in a gas mixture from the experiment on May 23rd, 2017.

in Figure 22. This figure shows that the increase of the concentration of ethane vapor in the gaseous mixture is in agreement with a change of the relative refractive index deduced from the rainbow measurement.

\section{Conclusion}

The rainbow refractometry laser diagnostic was developed and used to probe streams of fuel droplets in high-pressure conditions representative of transcritical conditions. Experiments were performed in a high-pressure optical facility specifically designed to inject free falling nearly-monodispersed droplets in the range of pressure 1-50 bar.

Experiments were initially performed using pure liquids (water and ethanol) that have low evaporation rate under elevated pressure (up to 50 bar). The behavior of their experimental refractive indices with pressure reveals a very good agreement with published results recorded in static liquid cells. These results demonstrate then very attractive properties for the rainbow refractometry, namely to measure with accuracy the refractive index of droplets in elevated pressures, results that have never been published in the open literature. Then, similar experiments were performed with ethane that is considered as a good working fluid for the study of fuel injection in transcritical conditions. Indeed, this fluid exhibits convenient thermodynamic critical properties easily accessible in laboratory experiments. The use of the rainbow refractometry on free falling ethane droplets with diameter of 100-150 $\mu \mathrm{m}$ under a pressure of 50 bar highlighted that the rainbow signals generated by ethane droplets were significantly affected by the gaseous species composition surrounding the droplets, in particular the gaseous ethane which is no longer negligible in these thermodynamic conditions. A development of a new methodology for processing the rainbow signals has generated a positive return on the simultaneous measurement of the ethane vapor concentration around the droplets as well as the absolute refractive index (i.e. temperature) and the size of liquid droplets. All these parameters will be then useful to improve our knowledge on evaporation processes when liquid fuel is injected in transcritical conditions.

To improve the accuracy of the measurements with the rainbow refractometry, further study must be done in the future to improve our methodology of measurement in order to acquire simultaneously and with a good accuracy the refractive index of the gaseous mixture surrounding the droplets (concentration of fuel vapor) as well as the refractive index of the liquid fuel (temperature and size of droplets). Therefore, the accuracy of measurements of the properties of droplets when transporting in a high evaporation transcritical environment, will be improved. One promising strategy would consist in recording the refractive index of the gaseous fluid surrounding the droplets transported in a high-pressure flowfield by using a light beam centered on the absorption frequency of the fuel and crossing the flowfield. Measurements of the light intensity before and after crossing the flowfield will be performed. By calculating the ratio of these signals (transmitted and reference signals), the determination of the fuel vapor concentration inside the chamber will be deduced. A combination of this optical measurement with measurements performed by the rainbow refractometry will finally provide a measurement of size and temperature of droplets as well as the fuel 
vapor concentration in high-pressure flow in which fuel droplets will produced.

\section{References}

1 Wensing M., Vogel T., Götz G. (2016) Transition of diesel spray to a supercritical state under engine conditions, Inter. J. Engine. Res. 17, 1, 108-119. doi: 10.1177/1468087415604281.

2 Poulter T.C., Ritchey C., Benz C.A. (1932) The effect of pression on the refractive index of refraction of paraffin oil and glycerine, Phys. Rev. 41, 3, 366.

3 Rosen J.S. (1947) The refractive indices of alcohol, water, and their mixtures at high pressures, J. Opt. Soc. Am. 37, 11, 932-938.

4 Waxler R.M., Weir C.E. (1963) Effect of pressure and temperature on the refractive indices of benzene, carbon tetrachloride, and water, J. Res. Natl. bur. Stand A Phy. Chem. 67/A, 163-171. doi: 10.6028/jres.067A.06.

5 Waxler R.M., Schamp H.W., Weir C.E. (1964) Effect of pressure and temperature upon the optical dispersion of benzene, carbon tetrachloride and water, J. Res. Natl. Bur. Stand. A 5, 489-498.

6 Stanley E.M. (1971) Refractive index of pure water for wavelength of 6328 and at high pressure and moderate temperature, J. Chem. Eng. Data 16, 4, 454-457.

7 Besserer G.J., Robinson D.B. (1973) Refractive indexes of ethane, carbon dioxide, and isobutane, J. Chem. Eng. Data 18, 2, 137-140.

8 Wang D.L., Yang K., Zhou Y. (2016) Measuring refractive index and volume under high pressure with optical tomography and light microscopy, Appl. Opt. 55, 9, 2435-2438.

9 Clifford T. (1999) Fundamentals of supercritical fluids, Oxford University Press, New York, USA.

10 Adam J.A. (2002) The mathematical physics of rainbows and glories, Phys. Rep. 356, 4, 229-365.

11 van de Hulst H.C. (1957) Light scattering by small particles, John Wiley \& Sons Inc., New York.

12 Lorenz L. (1890) Lysbevaegelsen i og uden for en af plane lysbolger belyst kulge, Vidensk. Selk. Skr 6, 1-62.

13 Mie G. (1908) Beiträge zur Optik Trüber Medien, speziell Kolloidaler Metallösungen, Ann. der Phys 25, 377-452.

14 Debye P. (1909) Der Lichtdruck auf Kugeln von beliebigem Material, Ann. der Phys. 30, 57-136.

15 Saengkaew S., Charinpanitku T., Vanisri H., Tanthapanichakoon W., Mees L., Gouesbet G., Gréhan G. (2006) Rainbow refractrometry: On the validity domain of Airy's and Nussenzveig's theories, Opt. Commun. 259, 7-13.
16 Nussenzveig H.M. (1969) High-frequency scattering by a transparent sphere. II. Theory of the rainbow and the glory, J. Math. Phys. 10, 125.

17 FMP Technology GmbH Fluid Measurement Projects (2011) Monodisperse droplet generator for industrial and university research applications. https://fmp-technology.com.

18 Marston P.L., Goosby S.G. (1985) Ultrasonically stimulated low-frequency oscillation and breakup of immiscible liquid drops: Photographs, Phys. Fluids 28, 1233-1242.

19 Roth N., Anders K., Frohn A. (1991) Refractive-index measurements for the correction of particle sizing methods, Appl. Opt. 30, 33, 4960-4965.

20 Roth N., Anders K., Frohn A. (1990) Simultaneous measurement of temperature and size of droplets in the micrometer rang, J. Laser App. 2, 1, 37-42.

21 Saengkaew S., Charinpanikul T., Laurent C., Biscos Y., Lavergne G., Gouesbet G., Gréhan G. (2010) Processing of individual rainbow signals, Exp. Fluids 48, 1, 111-119.

22 Van Beeck J.P.A.J., Giannoulis D., Zimmer L., Riethmuller M.L. (1999) Global rainbow thermometry for droplettemperature measurement, Opt. Lett. 24, 23, 1696-1698.

23 Wang J.J., Gréhan G., Han Y.P., Saengkaew S., Gouesbet G. (2011) Numerical study of global rainbow technique: sensitivity to non-sphericity of droplets, Exp. Fluids 51, 149-159.

24 Saengkaew S., Godard G., Blaisot B., Gréhan G. (2009) Experimental analysis of global rainbow technique: sensitivity of temperature and size distribution measurements to non-spherical droplets, Exp. Fluids 47, 839-848.

25 Verdier A., Santiago J.M., Vandel A., Saengkaew S., Cabot G., Gréhan G., Renou B. (2017) Experimental study of local flame structures and fuel droplet properties of a spray jet flame, Proc. Combust. Inst. 36, 2595-2602.

26 Ouboukhlik M., Saengkaew S., Fournier-Salaun M.C., Estel L., Gréhan G. (2015) Local measurement of mass transfer in a reactive spray for $\mathrm{CO}_{2}$ capture, Can. J. Chem. Eng. 93, 419-426.

27 Saengkaew S., Godard G., Gréhan G. (2018) Global Rainbow Technique: Temperature evolution measurements of supercold droplets, in: ICLASS 2018, 14th Triennial International Conference on Liquid Atomization and Spray Systems, Chicago, IL, USA, July 22-26, 2018.

28 Edlén B. (1966) Equation for the refractive index of air, Metrologia 2, 2, 71-80.

29 Schiebener P., Straub J.M.H., Levelt Sengers J.M.H., Gallagher J.S. (1990) Refractive index of water and steam as function of wavelength, temperature and density, J. Phys. Chem. Ref. Data 19, 3, 677-717. 